\title{
Watermarked Shadow Free Vop Sequences Using Hybrid Transforms
}

\author{
T. Vishnu Priya ${ }^{1}$, K. Gopi Suresh ${ }^{2}$, CH.Sandeep ${ }^{3}$, V. Siva Sai Kumar ${ }^{4}$ \\ , S. Rizwaan ${ }^{5}$ \\ ${ }^{I}$ (Asst. Professor in E.C.E dept, S.R.K.I.T/ JNTUK, INDIA) \\ ${ }^{2}$ (E.C.E, S.R.K.I.T/ Jntuk, India) ${ }^{3}$ (E.C.E, S.R.K.I.T/ Jntuk, India) \\ ${ }^{4}$ (E.C.E, S.R.K.I.T/ Jntuk, India) ${ }^{5}$ (E.C.E, S.R.K.I.T/ Jntuk, India)
}

\begin{abstract}
Today, detection and tracking is vital to many applications dealing with image sequences such as video surveillance like military, defense systems, law enforcements and cryptography. In object based coding, video frames are defined in terms of layers of Video Object Planes(VOP).With the development of modern technology more attention has been shifted towards spatial resolution for object recognition, mapping and image capabilities. Unexpected shadows effect its resolution. Removal of shadow from VOP's will assist the applications for comprehensive detection of activities. While transmitting video through unstructured network makes it vulnerable to many attacks. In order to protect the video content from attacks, digital watermarking is necessary. In this project, we implement to achieve an efficient algorithm for the watermarked VOP sequences by applying hybrid transformation techniques.
\end{abstract}

Keywords - Cryptography, Hybrid transformation, shadow, spatial resolution, VOP

\section{INTRODUCTION}

Computer Vision is concerned with the theory of building artificial systems that obtain information from images. The image data can have many forms such as video sequence, views from multiple cameras or multi-dimensional data from a medical scanner. There are many characteristics of computer vision which are considered to be relevant that are image processing/image analysis. These processes focused on 2D images and their transformations. Imaging which focuses on producing 2D \& 3D images. Machine vision focuses on manufacturing applications and Pattern Recognition. Typical tasks of computer vision are image recognition, motion, scene reconstruction, image restoration, image acquisition, pre-processing, feature extraction, detection and high-level processing. Organization of computer vision systems is application dependent. Video Surveillance is an important application of Computer Vision for an organization, from the security point of view. This is one application where an automated system can replace human beings. Detection and tracking is vital to many applications dealing with image sequences such as video surveillance like military, defense systems, law enforcements and cryptography. However, an unexpected shadow causes difficulties such as object merging, shape distortion and even loss of object [1].A shadow occurs when an object partially or totally occludes direct light from a source of illumination. In general, shadows can be divided into two major classes: self shadows and cast shadows. A self shadow occurs in the portion of an object which is not illuminated by direct light. A cast shadow is the area projected by the object in the direction of direct light. Cast shadows can be further classified into umbra and penumbra region, which is a result of multi-lighting and self shadows also have many sub-regions such as shading and inter reflection. Usually, the self shadows are vague shadows and do not have clear boundaries. On the other hand, cast shadows are hard shadows and always have a violent contrast to background. The difficulties in shadow detection arise because of two important visual features. First, since shadows typically differ from background, shadow points are detected as foreground objects. Second, shadows move in same motion as their objects casting them [1]. For these reasons, shadow detection and removal in images/videos is serious problem and has become an active research area. Any foreground object segmented out from a video which have a semantic meaning is called Video Object plane (VOP). An innate problem of VOP generation is that objects of interest are not homogeneous with respect to low level features such as color, intensity, texture and optical flow. Hence, conventional low-level segmentation algorithms will fail to extract required meaningful partitions. Hence automatic segmentation of VOPs is active research topic which has lot of challenging problems like shape changing, rotations, intra object motion, rigid parts, cluttered background, moving background and eliminating shadows etc., Nowadays, most of the data is saved in digital format. The advantage of saving the data in digital format is easy for creation, modification and distribution. The rise of Internet usage over the past few years has produced a diverse range of dynamic and highly interactive environment for this digital information exchange. However, the efficiency of information exchange and manipulation results the issue of copyright protection become increasingly significant. The possibility of 
unlimited copying of digital media without any loss of quality may cause the media producers and content providers a considerable financial loss. In order to protect the owner's copyright on their media watermarking technique was introduced. Watermarking is a technique of data hiding which embeds data into a digital signal. The new technology of digital watermarking has been advocated by many specialists as the best method to such multimedia copyright protection problems. It's expected that digital watermarking will have a wide span of above mentioned applications. The digital watermarking method to be effective it should be imperceptible and robust to common image manipulations like compression and other digital signal processing operations. In this project, we implemented automatic shadow removal algorithm in the first step, digital image watermarking algorithm based on Hybrid transforms in the next step and then extract the digital watermark by applying inverse of the above transform in the last step.

\section{Different Case Studies in Previous Work:}

\section{REVIEW OF THE PROJECT}

Case 1: Initially 5 percent noise level is added to the input VOP to obtain noisy VOP. The added noise is removed by applying CMF filter. Shadow has been removed from the VOP with their proposed algorithm. Watermark is added to the resultant VOP. The watermark is extracted from the VOP. To know the effect, quality checking metrics have been used i.e. PSNR value.

Case 2: In the second test case, noise is added to input VOP and it is removed prior to shadow elimination. After elimination of shadow watermarking is done and next water mark is extracted.

Case 3: In this test case, initially shadow has been eliminated from the input VOP. Addition of noise and reduction of noise is performed in subsequent steps. A watermark is added to the resultant VOP and next water mark is extracted.

Case 4: Initially, shadow is eliminated from the input VOP. The resultant shadow eliminated VOP, 5 percent level of noise is added which is then reduced. For authentication, a watermark is embedded into the resultant VOP and extracted.

Case 5: The experimental setup of test case 4 is similar to setup of test case5. Some modules have been interchanged to have complete view of results of different tests. Initially, shadow is eliminated from input VOP. A watermark is embedded by using DWT watermarking for authentication. To study noise effect, some noise is inserted which is then tried to reduce by applying noise removal smoothing filter on the resultant VOP. The watermarks are extracted then to discover the distortion occurred to them.

The above cases does not yield better results so we proposed a method in which noise is not added and removed, In the watermarking we use hybrid Transforms instead of applying single transform i.e. either DCT or DWT. Due to this more security is available.

Proposed Method:

The block diagram of proposed method as shown below

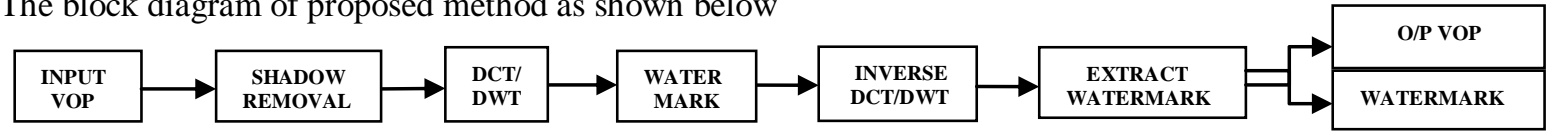

Fig.1: Block Diagram of Proposed Method

\section{SHADOW REMOVAL}

Shadow:

A shadow is an area where direct light from a light source cannot reach due to obstruction by an object. It occupies all of the space behind an opaque object with light in front of it. The cross-section of a shadow is a two-dimensional silhouette or reverses projection of the object blocking the light. Sunlight causes many objects to have shadows at certain times of the day. The angle of the sun and its apparent height in the sky causes a change in the length of shadows. Low-angles create longer shadows.

Shadow occurs when object partially or totally occludes light from light source. Shadow in image reduces the reliability of many computer vision algorithms and often degrades the visual quality of images that we cannot recognize the original image of a particular object. Shadows are basically classified into two types namely 1) Static shadows 2) Moving shadows. In Static shadows the VOP sequences are stationary with respect to that of the object under observation. Moving shadows indicates shadows motion with respect to the object that is moving. It is easy to eliminate static shadow by background subtraction method but the moving shadow is moving with the movement objects, so the background subtraction cannot remove the moving shadow.

Here are our basic assumptions discussed in this paper. First the illumination image is spatially smooth. Second there is no change in the texture inside the shadow region and finally in the shadow regions, the illumination image is close to be constant. Pixels inside shadow regions have different colors because of the reflectance image not the illumination one. 


\section{Color transformation shadow removal:}

a) Color transformation:

The RGB-based color images are converted into HSV color space. In HSV color space, shadow regions hold some special properties (color and optical properties) which can be used for shadow segmentation.

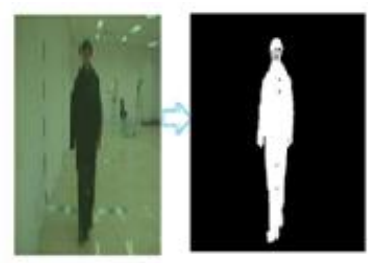

b) Shadow segmentation:

Fig.2: RGB to HSV Color Space Image

Based on these particular properties of shadows, the normalized saturation-value difference index is constructed to identify shadows. NSVDI is calculated by subtracting the value component from the saturation component for each image pixel. In NSVDI images, shadow regions are segmented by a threshold.

c) Shadow compensation:

Histogram matching method is used to adjust the hue, saturation and value component in segmented shadow regions respectively, by matching histogram with the local surroundings around each shadow region. Then, the result images are converted back to RGB color space.

Methodology for Shadow Removal:

Foreground Detection:

a) Background Reference image:

A background reference image is required for the process of shadow removal. It will be used to as reference to every new frame which will result in the outline of objects and their shadows. I used the first frame of every video as the background reference image.

b) Background subtraction:

Background subtraction is a widely used method in Computer Vision for separating or segmenting out the foreground objects from the background of a video. The foreground objects are defined to be the parts of the image that changes and the background is made out of the pixels that stay relatively constant.

It is the first step in extraction. The difference between the current frame and the background shows the dynamic context. The Otsu threshold method is used to transform the different images into a binary map. In binary map, there is lot of influential noises. For this situation, this paper uses a binary morphological algorithm to filter the region which contains the number of white pixels less than the default threshold $\mathrm{N}$ in the 8 bordering regions, thereby removing noise influence. After removing noise from the binary map, it can be seen that a lot of noise has been filtered out, but the bike on the left of the lane line and the vehicle in the far right lane line still have some influence. To remedy this, this project extracts auxiliary lane line positions to eliminate the influence.

In order to ensure the integrity of the object, expansion of binary map processing should be carried out. Image expansion can effectively repair the holes and smooth the edges of objects. Then, it is used to mark object areas and to compute the areas of every separate block in order to transform them into block diagrams for object extraction.

Background subtraction alone cannot effectively remove shadow influences because object and background gray levels are too similar (the profile extracted by background subtraction is incomplete when object and background have similar gray levels) so the vehicle profile cannot meet the needs of the illegal lane changing detection system. So, a second step of object edge extraction is put forward.

In this project we mainly used the background subtraction algorithm and thresholding described in [2]. However, for the first step, we assume that we have the background image that has been registered initially. Thus, we do not need to do background registration. Next, since we assume the illumination is high, threshold with proper values gives the required effects.

First we do absolute frame difference between subsequent grabbed frames and then we threshold the difference images. To suppress the shadow, threshold values is chosen high.

The approach taken to identifying foreground regions is to:

1. Construct a model of the background scene by sampling the scene when no people or other relevant objects are present.

2. Compare new images of the scene to this background model and determine any discrepancies; these are considered foreground regions.

3. Update the background model as each new image is collected.

The background model allows for a mismatch between the locations of pixels in successive images, i.e., pixel shift. For each pixel, a window around the previous position of the pixel is searched to determine the new 
location of the pixel. This stabilizes the image, countering for example, the results of a shaking camera. Note that the foreground region generated by a person in the image will consist of both the person and his/her shadow. The last step in the background subtraction is to eliminate the shadow region. Shadows are patches of a background region in which the color value is the same, but the intensity is darker.

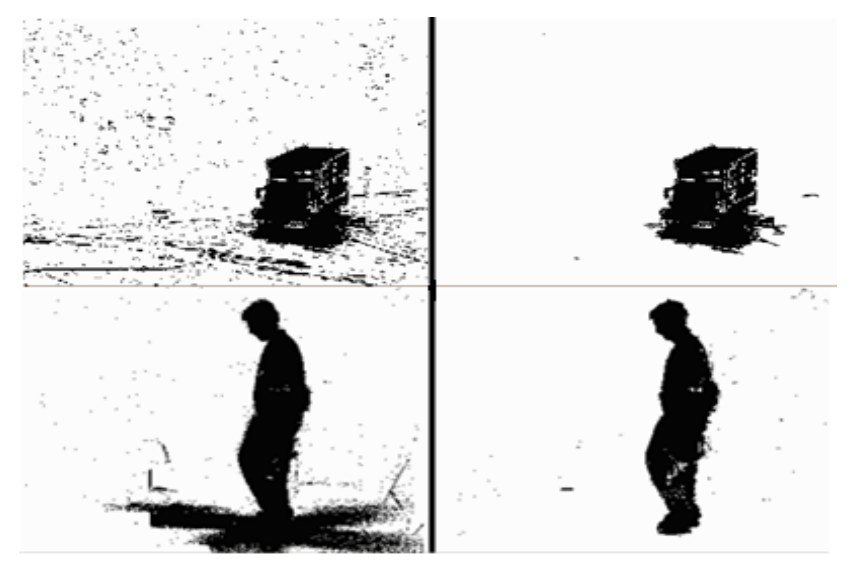

Fig.3: Stabilization (left) and Shadow Elimination (right)

The left images are unprocessed background subtraction, and the right images are the processed background subtraction.

\section{c) Normalizing the RGB factor in each frame:}

In this we have done pre-processing for the background reference image and also for the subsequent frame of the given video. This is done to remove the change of intensity in any frame. Converting an RGB image into normalized RGB removes the effect of intensity variations.

This takes places as follows

1. First will convert the image to double for accurate calculation.

2. After converting the frame to double will normalize the each color red, green and blue of a frame so that we will reduce the effect of change in intensity in a frame. We will normalize each $R, G, B$ part individually.

In this project we performed the normalization of RGB color space model for frame and after that it subtracts each frame of the video from the background frame

After that we applied the threshold on each frame so that we can get a shadow free image in distorted frame where we will just show the moving object. Threshold limit for the image is done by

RGB to HSV conversion formula:

The $R, G, B$ values are divided by 255 to change the range from $0-255$ to $0-1$ :

$$
\begin{array}{lll}
\mathrm{R}^{\prime}=\mathrm{R} / 255 & \mathrm{G}^{\prime}=\mathrm{G} / 255 & \mathrm{~B}^{\prime}=\mathrm{B} / 255 \\
C_{\text {max }}=\max \left(R^{\prime}, G^{\prime}, B^{\prime}\right) & C_{\text {min }}=\min \left(R^{\prime}, G^{\prime}, B^{\prime}\right) & \Delta=C_{\text {max }}-C_{\text {min }}
\end{array}
$$

$\mathrm{H}=\left\{\begin{array}{l}60^{\circ} \times\left(\frac{G^{\prime}-B^{\prime}}{\Delta} \bmod 6\right), C_{\max }=R^{\prime} \\ 60^{\circ} \times\left(\frac{B^{\prime}-R^{\prime}}{\Delta}+2\right), C_{\max }=G^{\prime} \\ 60^{\circ} \times\left(\frac{R^{\prime}-G^{\prime}}{\Delta}+4\right), C_{\max }=B^{\prime}\end{array}\right.$

Saturation calculation:

$$
s=\left\{\begin{array}{c}
0, \quad \Delta=0 \\
\frac{\Delta}{C_{\max }}, \Delta<>0
\end{array}\right.
$$

Value calculation:

$$
\mathrm{V}=C_{\text {max }}
$$

Table: The Data Contrast Between the Body and the Background 


\begin{tabular}{|c|l|l|l|l|l|l|}
\hline & \multicolumn{2}{|l|}{$(284,154)$} & \multicolumn{2}{c|}{$(294,154)$} & $(306,159)$ \\
\hline Pixel & Body & Back-ground & Body & Back-ground & Body & Back-ground \\
\hline R & 78 & 125 & 81 & 126 & 151 & 118 \\
\hline G & 37 & 123 & 32 & 124 & 81 & 122 \\
\hline B & 43 & 126 & 36 & 129 & 81 & 123 \\
\hline$C_{r}$ & .494 & .334 & .544 & .333 & .482 & .325 \\
\hline$C_{g}$ & .234 & .329 & .215 & .327 & .259 & .336 \\
\hline$C_{b}$ & .272 & .337 & .242 & .340 & .259 & .339 \\
\hline$\Delta_{r}$ & +0.160 & \multicolumn{2}{|c|}{+0.211} & +0.157 \\
\hline$\Delta_{b}$ & -0.095 & -0.112 & -0.077 \\
\hline$\Delta_{g}$ & -0.065 & -0.098 & -0.080 \\
\hline
\end{tabular}

From the below table we analysis the threshold so that we can get the shadow free image and from the above table we can see that maximum change is in the Red color and the maximum change is around 0.211. This is for the particular video. So in general we can make it more flexible and can adjust it to the general value for the entire image. So finally I taken the threshold as 0.35

Threshold $=0.35$ (Depends on the video file)

Threshold of 0.35 is applied to get the shadow free image. Threshold should be adjusted for other video files.

$\mathrm{P}(\mathrm{P}<0.35)=0$;

$\mathrm{P}(\mathrm{P}>=0.35)=1$;

By performing the above function we will get a shadow free image with the background and just we will see the moving object. Finally we got a shadow free image, but we need to reconstruct it so morphological operation is applied on this image so that we can get original video with just the detection of moving object.

\section{Morphological Operation:}

Morphology is a broad set of image processing operations that process images based on shapes. Morphological operations apply a structuring element to an input image, creating an output image of the same size. In a morphological operation, the value of each pixel in the output image is based on a comparison of the corresponding pixel in the input image with its neighbours. By choosing the size and shape of the neighbourhood, you can construct a morphological operation that is sensitive to specific shapes in the input image. We need exact boundary of the objects and their shadows. For this we applied background subtraction on gray scale image of the each frame of the video.
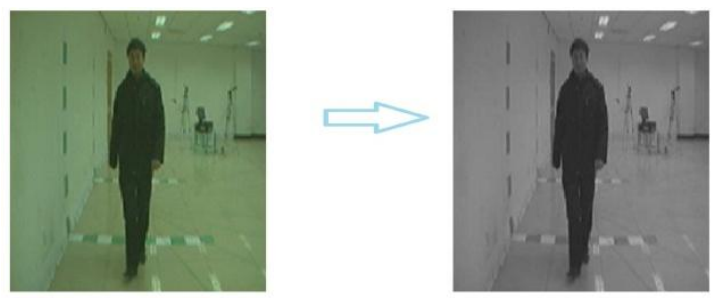

Fig 4: RGB to grey scale image

Convert the RGB image into the gray scale so that we can apply the background subtraction on it. For this process we converted the background reference image as well as the current frame to gray scale and then performed the operation on the gray scale image of the video sequence.

Dilation adds pixels to the boundaries of objects in an image, while erosion removes pixels on object boundaries. The number of pixels added or removed from the objects in an image depends on the size and shape of the structuring element used to process the image. After the above step we will do dilation on the above frame and well use infill to fill out the holes in a bound area of an image.

\section{INTRODUCTION:}

\section{WATERMARKING}

General Definition of Watermark:

A watermark is a recognizable image or pattern in paper that appears as various shades of lightness/darkness when viewed by transmitted light (or when viewed by reflected light, atop a dark 
background), caused by thickness or density variations in the paper. Watermarks have been used on postal stamps, currency, and other government documents to discourage counterfeiting.

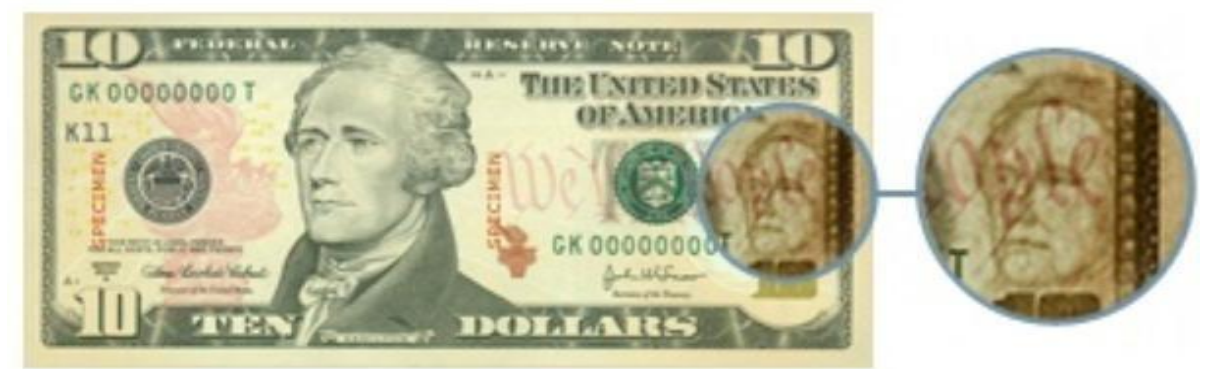

What is Digital Watermarking?

Fig.5: Watermarked currency

Digital watermarking can be defined as the process of embedding a certain piece of information (known as watermark) into multimedia content including text documents, images, audio or video streams, such that the watermark can be detected or extracted later in order to provide copyright protection to the data and prevent illegal copying.

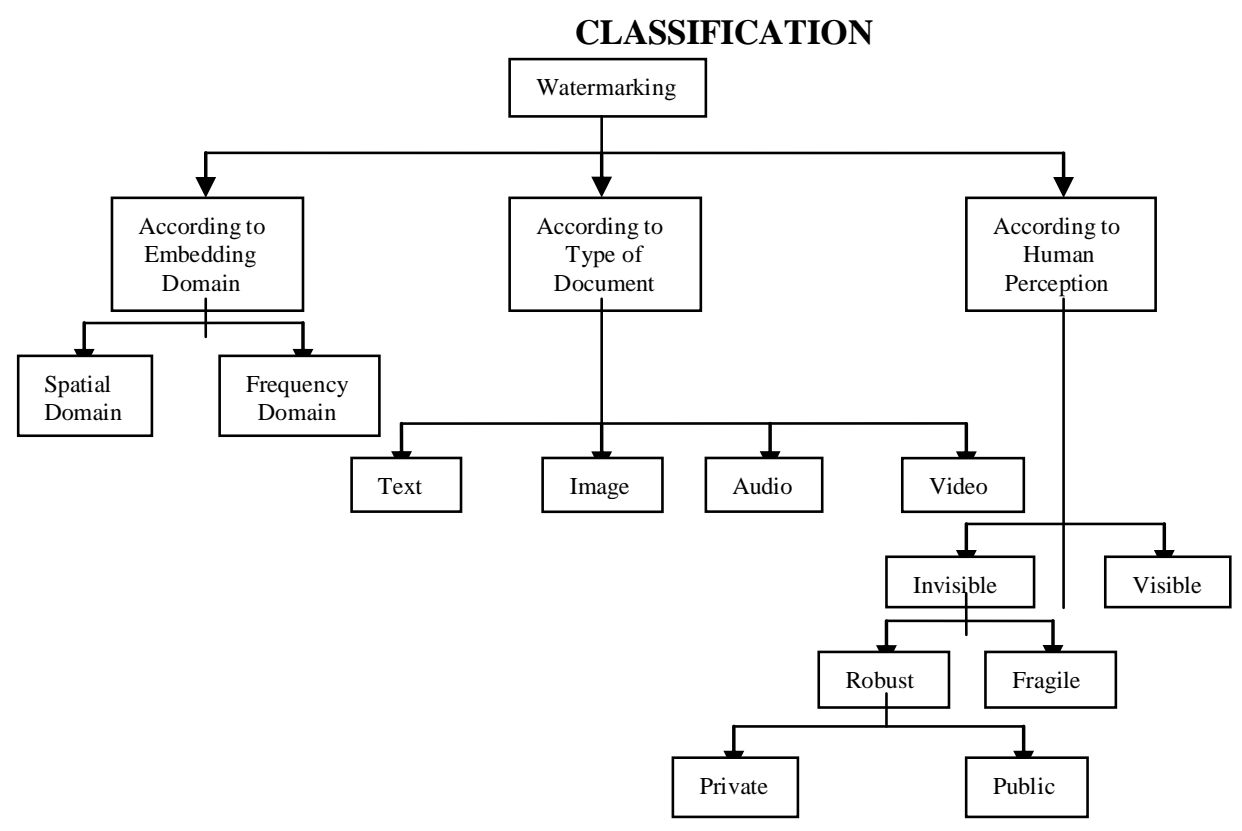

Fig.6: Watermarking Classification

Techniques According To Working Domain:

Spatial Vs Frequency domain:

Spatial watermarking is easy to implement from computational point of view but too fragile to withstand large varieties of external attacks. Frequency or transformed domain approach offers robust watermarking but in most cases implementation needs higher computational complexity. Moreover the transform domain technique is global in nature (global within the block in block-based approach) and cannot restrict visual degradation of the cover image. But in spatial domain technique, degradation in image quality due to water marking could be controlled locally leaving the region of interest unaffected.

The watermarks that are embedded in spatial domain will easily be removed by common filtering process. Besides that, some of the spatial domain watermarking scheme are unable to detect the embedded watermark when the watermarked image underwent geometrical distortion like additional of noise.

Applications of Watermarking:

Copyright Protection \& Owner identification:

To protect its intellectual property, the data owner can embed a watermark representing copyright information of his data. This application can be a really helpful tool in settling copyright disputes in court. It is probably the most widely spread use of digital images watermarking and it is also the application we have worked on in the present project. 


\section{Broadcast monitoring:}

In order to help the automated identification of broadcasted programs, original watermarks can be inserted in any type of data to be widely broadcasted on a network. It could assure that advertisers received the airtime they have paid for or make certain that musicians' property is not rebroadcast by pirate stations (or at least, if so, that it can be detected).

Data Authentication:

Fragile watermarks are used to detect any corruption of an image or any other type of data. If the watermark is detected, the data is genuine, if not the data has been corrupted and cannot be considered.

Data Hiding (Covert Communications):

The transmission of private data is probably one of the earliest applications of watermarking. As one would probably have already understood, it consists of implanting a strategic message into an innocuous one in a way that would prevent any unauthorized person to detect it.

\section{Requirements of Watermarking:}

To achieve maximum protection of intellectual property with watermarked media, several requirements must be satisfied

Undeletable:

The watermark must be difficult or even impossible to remove by a hacker, at least without obviously degrading the host signal.

Statistically Undetectable:

A pirate should not be able to detect the watermark by comparing several watermarked signals belonging to the same author.

Unambiguous:

Retrieval of the watermark should be unambiguously identify the owner, and the accuracy of identification should degrade gracefully in the face of attacks

Security:

The security requirement of a watermarking system can differ slightly depending on the application. Watermarking security implies that the watermark should be difficult to remove or alter without damaging the host signal. As all watermarking systems seek to protect watermark information, without loss of generality, watermarking security can be regarded as the ability to assure secrecy and integrity of the watermark information, and resist malicious attacks.

Robustness:

The watermark should be surviving the lossy compression techniques like JPEG, which is commonly used for transmission and storage. The watermark should be retrievable even if common signal processing operation are applied, such as signal enhancement, geometric image operations, noise, filtering, and etc.

\section{HYBRID TRANSFORMS}

\section{Discrete Cosine Transformation (DCT):}

DCT like a Fourier Transform, it represents data in terms of frequency space rather than an amplitude space. This is useful because that corresponds more to the way humans perceive light, so that the part that are not perceived can be identified and thrown away. DCT based watermarking techniques are robust compared to spatial domain techniques. Such algorithms are robust against simple image processing operations like low pass filtering, brightness and contrast adjustment, blurring etc. However, they are difficult to implement and are computationally more expensive. At the same time they are weak against geometric attacks like rotation, scaling, cropping etc. DCT domain watermarking can be classified into Global DCT watermarking and Block based DCT watermarking. Embedding in the perceptually significant portion of the image has its own advantages because most compression schemes remove the perceptually insignificant portion of the image.

The discrete cosine transforms is a technique for converting a signal into elementary sum of sinusoids of varying magnitudes and frequencies. With an input image, X, the DCT coefficients for the transformed output image, Y, are computed according to Eq. 6 shown below. In the equation, X, is the input image having $\mathrm{N} \times \mathrm{M}$ pixels, $\mathrm{X}(\mathrm{m}, \mathrm{n})$ is the intensity of the pixel in row $\mathrm{m}$ and column $\mathrm{n}$ of the image, and $\mathrm{Y}(\mathrm{u}$, $v)$ is the DCT coefficient in row $u$ and column $v$ of the DCT matrix.

$$
Y(u, v)=\sqrt{\frac{2}{M}} \sqrt{\frac{2}{N}} \alpha_{u} \alpha_{v} \sum_{\mathrm{u}=0}^{\mathrm{M}-1} \sum_{\mathrm{v}=0}^{\mathrm{N}-1} \mathrm{X}(\mathrm{m}, \mathrm{n}) \cos \frac{(2 m+1) u \pi}{2 M} \cos \frac{(2 n+1) v \pi}{2 N}
$$

Where

$$
\begin{aligned}
& \alpha_{u}=\left\{\begin{array}{rr}
\frac{1}{\sqrt{2}} & u=0 \\
1 & u=1,2, \ldots, N-1
\end{array}\right. \\
& \alpha_{v}=\left\{\begin{array}{rr}
\frac{1}{\sqrt{2}} & v=0 \\
1 & v=1,2, \ldots, N-1
\end{array}\right.
\end{aligned}
$$

The image is reconstructed by applying inverse DCT operation according to Eq. 7 : 
$X(m, n)=\sqrt{\frac{2}{M}} \sqrt{\frac{2}{N}} \sum_{\mathrm{u}=0}^{\mathrm{M}-1} \sum_{\mathrm{v}=0}^{\mathrm{N}-1} \alpha_{u} \alpha_{v} \mathrm{Y}(\mathrm{u}, \mathrm{v}) \cos \frac{(2 m+1) u \pi}{2 M} \cos \frac{(2 n+1) v \pi}{2 N}$

The popular block-based DCT transform segments an image non-overlapping block and applies DCT to each block. This result in giving three frequencies Sub-bands: low frequency sub-band, mid-frequency sub-band and high frequency sub-band. DCT-based watermarking is based on two facts. The first fact is that much of the signal energy lies at low-frequencies sub-band which contains the most important visual parts of the image. The second fact is that high frequency components of the image are usually removed through compression and noise attacks. The watermark is therefore embedded by modifying the coefficients of the middle frequency sub-band so that the visibility of the image will not be affected and the watermark will not be removed by compression.

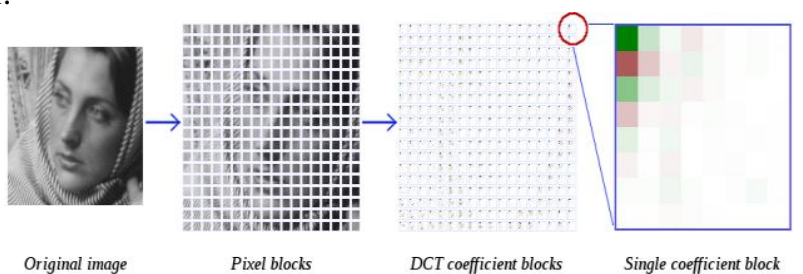

Fig.7: Mechanism of DCT Transform

Advantages:

1) DCT domain watermarking is comparatively much better than the spatial domain encoding since DCT domain watermarking can survive against the attacks such as noising, compression, sharpening, and filtering.

2) It uses JPEG compression method to apply DCT watermarking as a parameter. One may use different parameters related to image processing, and these parameters might provide equal or even stronger robustness against various attacks based on image processing [7].

3) Discrete cosine Transform (DCT), where pseudorandom sequences, such as M sequences, are added to the $\mathrm{DCT}$ at the middle frequencies as signatures.

\section{Discrete Wavelet Transformation (DWT):}

The Discrete Wavelet Transform (DWT) [5] is currently used in a wide variety of signal processing applications such as in audio and video compression, removal of noise in audio, and the simulation of wireless antenna distribution. Wavelets have their energy concentrated in time and are well suited for the analysis of transient, time-varying signals. Since most of the real life signals encountered are time varying in nature, the Wavelet Transform suits many applications very well. Wavelets are special functions in a form analogous to Sines and cosines in Fourier analysis, are used as basal functions for representing signals. For 2-D images, applying DWT corresponds to processing the image by 2-D filters in each dimension. The filters divide the input image into four non-overlapping multi-resolution sub-bands LL1, LH1, HL1 and HH1. The sub-band LL1 represents the coarse-scale DWT coefficients while the sub-bands LH1, HL1 and HH1 represent the finescale of DWT coefficients. To obtain the next coarser scale of wavelet coefficients, the sub-band LL1 is further processed until some final scale $\mathrm{N}$ is reached. When $\mathrm{N}$ is reached we will have $3 \mathrm{~N}+1$ sub-bands consisting of the multi-resolution sub-bands $\mathrm{LL}_{N}$ and $\mathrm{LH}_{X}, \mathrm{HL}_{X}$ and $\mathrm{HH}_{X}$ where $\mathrm{x}$ ranges from 1 until $\mathrm{N}$ [6].

Due to its excellent Spatio-frequency localization properties, the DWT is very suitable to identify the areas in the host image where a watermark can be embedded effectively. In particular, this property allows the exploitation of the masking effect of the human visual system such that if a DWT coefficient is modified, only the region corresponding to that Coefficient will be modified. In general most of the Image energy is concentrated at the lower frequency Sub-bands $\mathrm{LL}_{X}$ and therefore embedding watermarks in these sub-bands may degrade the image significantly. Embedding in the low frequency sub- bands, however, could increase robustness significantly. On the other hand, the high frequency sub-bands $\mathrm{HH}_{X}$ include the edges and textures of the image and the human eye is not generally sensitive to changes in such sub-bands. This allows the watermark to be embedded without being perceived by the human eye. The compromise adopted by many DWT-based watermarking algorithm, is to embed the watermark in the middle frequency sub-bands $\mathrm{LH}_{X}$ and $\mathrm{HL}_{X}$ where acceptable performance of imperceptibility and robustness could be achieved. 

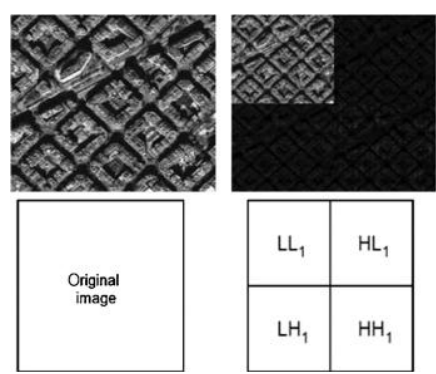
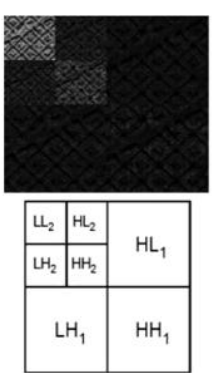

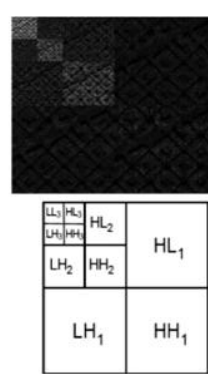

Fig.8: Level decompositions of DWT Transform

\section{Advantages:}

1) The watermarking method has multi resolution characteristics and is hierarchical. It is usually true that the human eyes are not sensitive to the small changes in edges and textures of an image but are very sensitive to the small changes in the smooth parts of an image. With the DWT, the edges and textures are usually to the high frequency sub-bands, such as HH, LH and HL etc. Large frequencies in these bands usually indicate edges in an image [7].

2) The watermarking method robust to wavelet transform based image compressions, such as the embedded zero-tree wavelet (EZW) image compression scheme, and as well as to other common image distortions, such as additive noise, rescaling/stretching, and half toning. This is advantage over DCT.

In this project, we will describe a digital image watermarking algorithm based on combining two transforms DWT and DCT. Watermarking is done by altering the wavelet coefficients of carefully selected DWT subbands, followed by the application of the DCT transform on the selected sub-bands:

\section{HYBRID TRANSFORMS ALGORITHM:}

In this algorithm [6] first of all the DWT of image is taken which gives us 4 different components of the image out of which I am embedding the watermark in either horizontal or vertical component by taking its DCT. After embedding watermark we are taking inverse DCT and inverse DWT respectively to get original view of image back.

Steps in Embedding Watermarking using Hybrid Transforms:

Step 1: Apply DWT to decompose the cover host image into four non-overlapping multi-resolution sub bands: LL, HL, LH, and HH.

Step 2: Apply DCT to each block in the chosen sub-band

Step 3: Re-formulate the grey-scale watermark image into a vector of zeros and ones.

Step 4: Generate two uncorrelated pseudorandom sequences. One sequence is used to embed watermark bit 0 (PN_0) and the other sequence is used to embed the watermark bit 1 (PN_1). (Number of elements in each of the pseudorandom sequences must be equal to the number of mid-band elements of the DCT-transformed DWT sub-bands.)

Step 5: Embed the pseudorandom sequences, PN_0 and PN_1, with a gain factor alpha $(\alpha)$, in the DCT transformed $8 \times 8$ blocks of the selected DWT sub-bands of the host image.

Step 6: Embedding is not to all coefficients of the DCT block, but only to the mid-band DCT coefficients. If we donate $\mathrm{X}$ as the matrix of the mid-band coefficients of the DCT transformed block, then embedding is done as follows:

If the watermark bit is 0 then

Otherwise,

$$
X^{\prime}=X+\alpha * P N \_0
$$

If the watermark bit is 1 then,

$$
X^{\prime}=X+\alpha * P N_{-} 1
$$

Step 7: Apply inverse DCT (IDCT) to each block after its mid-band coefficients have been modified to embed the watermark bits as described in the previous step.

Step 8: Apply the inverse DWT (IDWT) transform on the DWT transformed image, including the modified subband, to produce the watermarked host image. 


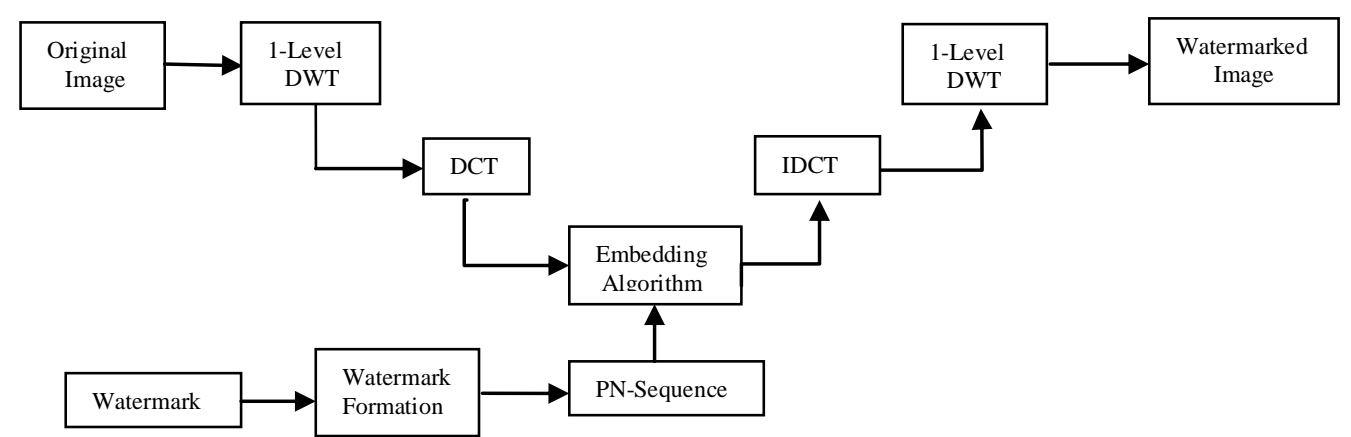

Fig.9: Combined DWT-DCT Watermark Embedding Procedure

The watermark extraction procedure is depicted in Fig. 5.4, and described in details in the following steps. The combined DWT-DCT algorithm is a blind watermarking algorithm, and thus the original host image is not required to extract the watermark.

Steps in Extraction of Watermark Using Hybrid Transforms:

Step 1: Apply IDCT to decompose the watermarked image into non-overlapping multi-resolution sub band: LL. Step 2: Apply IDWT to chosen sub-band (LL), and extract the VOP.

Step 3: Reconstruct the watermark using the extracted watermark bits, and compute the similarity between the original and extracted watermark and VOP.

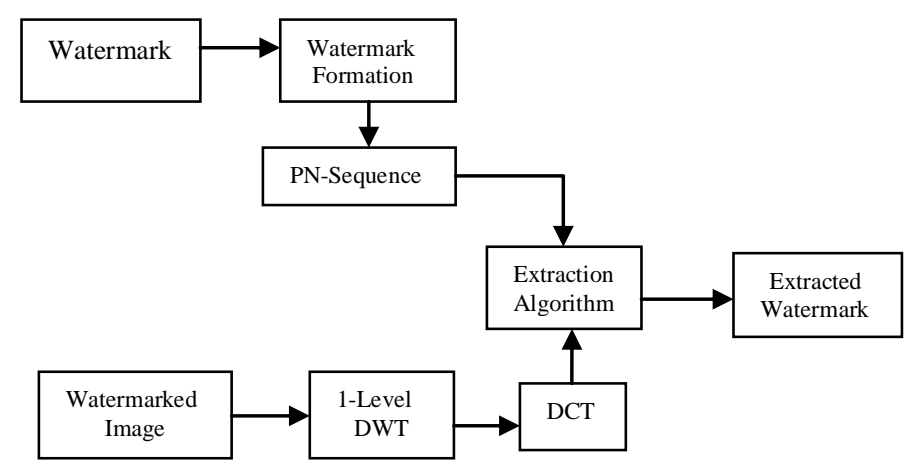

Fig.10: Combined DWT-DCT Watermark Extraction Procedure

VI. RESULT

original trame

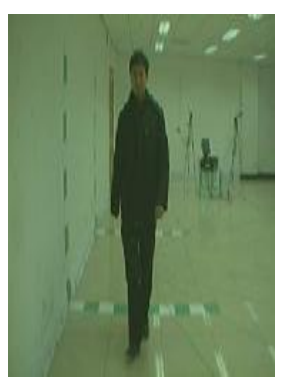

Fig.11: Original Frame and Foreground VOP

foreground VOP

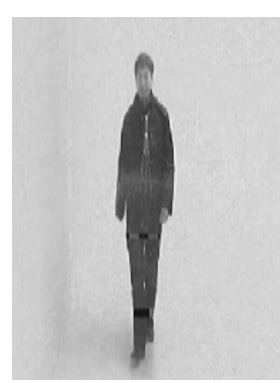

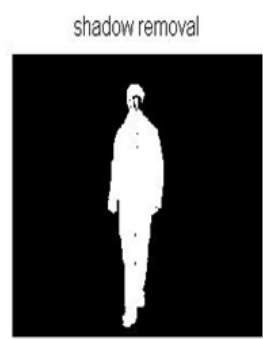

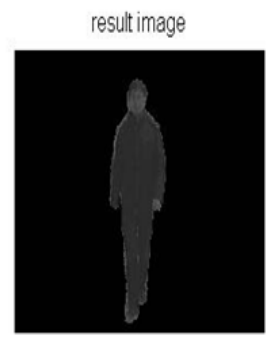

Fig.12: Shadow Removal and Result Image 

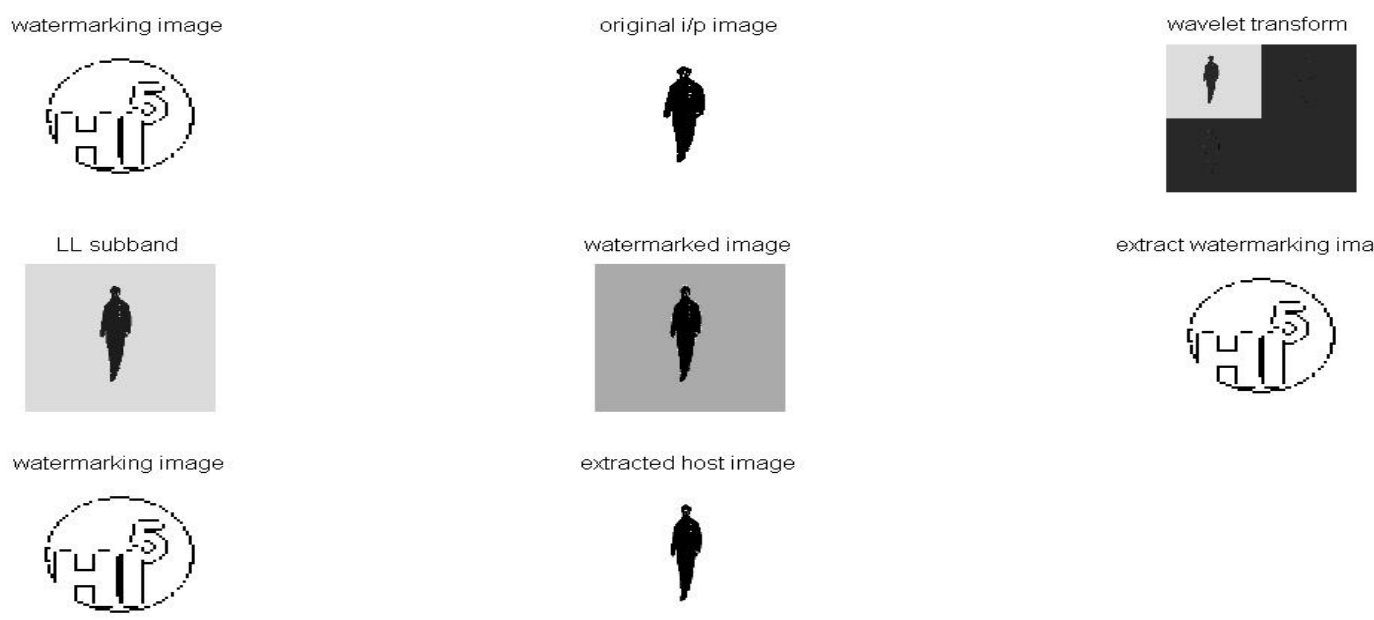

extract watermarking image

extracted host image
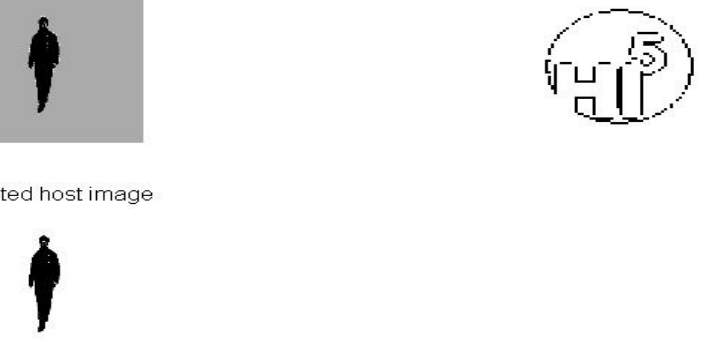

Fig.13: Embedding And Extract Watermark Image

VII. CONCLUSION

In this project we implemented RGB color space model for shadow removal from VOP. This algorithm gives good results for the VOP and also computation efficient. In this shadow and the moving object are discriminated by cascading two estimators which use the optical properties of color.

The Discrete Wavelet Transform (DWT) and the discrete cosine transform (DCT) have been successfully applied in many digital image watermarking applications. In this project, we combined DWT with another equally powerful transform i.e. DCT to improve the performance. The combined DWT-DCT watermarking algorithm's imperceptibility performance was better than the performance of the DWT-Only approach. Moreover, the improvement in robustness brought by the combined DWT-DCT algorithm was considerably high. Hence we obtained the super-resolved watermarked VOP sequences using Hybrid transforms (combination of DCT and DWT).

\section{FUTURE ASPECTS}

In future this method is applicable for color videos due to the application in surveillance. For robust authentication purpose this method is also used along with PN sequence. In this project the watermark is added in a particular VOP but the automatic process of watermarking of video may give better result for authentication.

\section{REFERENCES}

[1] Andrea Prati, Ivana Mikic, Mohan M. Trivedi and Rita Cucchiara. Detecting Moving Shadows: Algorithms and Evaluation. IEEE Transactions on Pattern Analysis and Machine Intelligence, vol.25, no. 7, July 2003.

[2] N. Otsu, "A Threshold Selection Method from Gray-Level Histogram", IEEE Trans. Systems Man, and Cybernetics, Vol. 9, pp. 62-66, 1979.

[3] Elgammal, R. Duraiswami, and L. S. Davis, "Efficient computation of kernel density estimation using fast gauss transform with applications for segmentation and tracking," Proc. IEEE 2nd Int. Workshop Statistical and Computational Theories of Vision, July 2001.

[4] Stefan Katzenbeisser and Fabien A. P. Petitcolas, "Information Hiding Techniques for Steganography and Digital Watermarking," Artech house, Computer security series, pp.15-23, 97-109, 2000.

[5] Guangmin Sun and Yao Yu. DWT based Watermarking Algorithm of Color Images. IEEE Conference on Industrial Electronics and Applications, ICIEA, pp.1823-1826, 2007.

[6] Journal of Computer Science 3(9):740-746, 2007ISSN 1549-3636 @ 2007 Science Publications

[7] Darshana Mistry / (IJCSE) International Journal on Computer Science and Engineering Vol. 02, No. 09, 2010, 2905-2909. 